\title{
Forensic analysis of braking classification based on acceleration, jerk,
} and velocity data

Bayu Erfianto*1, Andrian Rahmatsyah ${ }^{2}$

Department of Information Technology, Universitas Telkom, Indonesia ${ }^{1}$

Department of Informatics, Universitas Telkom, Indonesia ${ }^{2}$

\section{Article Info}

Keywords:

Acceleration, Jerk, Velocity, Brake, Forensic Analysis

\section{Article history}

Received: July 05, 2021

Accepted: July 26, 2021

Published: August 31, 2021

Cite:

Erfianto, B., \& Rahmatsyah, A. (2021).

Forensic Analysis of Braking Classification

Based on Acceleration, Jerk, and Velocity

Data. Kinetik: Game Technology, Information

System, Computer Network, Computing,

Electronics, and Control, 6(3).

https://doi.org/10.22219/kinetik.v6i3.1284

*Corresponding author

Bayu Erfianto

E-mail address:

erfianto@telkomuniversity.ac.id

\begin{abstract}
Nowadays, four-wheeled vehicles are equipped with an event data recorder (EDR) device to record sensors data. With advances in-memory technology, EDR provides evidence for forensic analysis after an accident happens, that uses information technology to facilitate forensic analysis to provide complete and valuable results using digital investigations. Several types of research have been conducted to reconstruct accidents from forensic data and Fuzzy Logic is an alternative method for classifying crash data taken from the accelerometer due to less complexity of implementation. Vehicle braking data is one of the most important evidence for digital investigation, since braking is a complex process determined by many factors, such as the condition of the vehicle, road construction, and the driver's physiological condition. However, the existing digital investigation still process vehicle speed, deceleration, and varia- tion time of deceleration (known as a jerk) in separated manner to determine braking distance, driver response time, and braking category. The problem identified in this paper is how to use deceleration, velocity, and jerk to categorize the braking evidence forensic analysis. In this paper, forensic analysis is limited to produce forensic evident of braking events based on the collected data. The contribution of this paper is to propose a braking detection model by combining acceleration, speed, and jerk data into a Fuzzy Inference System. As a result, a forensic analysis of braking data can better understand the braking maneuvers, which can be further developed to identify the cause of the accident and provide recommendations on which actions to include in future analyses.
\end{abstract}

\section{Introduction}

Dobromirov in his research [1] defines digital forensics as the science that identifies, extracts, analyzes, and presents dig- ital evidence that has been stored in digital electronic storage devices for use in the court of law. Regarding this definition, there are several stages in digital forensics, including incident detection, physical investigation, and digital investigation. Several four-wheeled vehicles today are equipped with an event data recorder (EDR) device. It initially was used to record sensor data to see whether the airbag is in the active status that caused accidents among fourwheeled vehicles [2]. At this moment, some of the sensor status, however, can also be recorded by the EDR, such as Flight Data Recorder. Thus, EDR can be used as evidence for forensic analysis in the event of an accident on the vehicle. Hoppe in his research [3] discussed vehicle accident forensic using information technology in it to obtain the evidence of vehicle forensic parameters and to save them on a memory card. With the advances in technology of memory for digital investigation, the uniformity of data storage media (memory) and data formats can be carried out to facilitate a forensic analysis to provide more complete and useful results [4].

Recently [5] developed an embedded sensor system to measure comfort in public transportation system. This researcher used a triaxial accelerometer and GPS receiver, and SDCard storage. This researcher also developed an embedded sensor device using a tri-axial accelerometer, GPS receiver, and SDCard to record all sensor data. Other researchers such as Nguyen [6], Sim [7], and Jhou [8] in their research discussing the On-Board Diagnostic II (OBD-II) system, which is designed to monitor the status of the vehicle contin- uously. Research conducted by [9] uses OBD data (onboard device) and an accelerometer that is installed separately from the OBD system to perform EDR data recording. OBD-II is a set of standards for implementing a computer-based system to monitor the performance of several major engine components and several other vehicle parameters, where OBD II has become the SAE standard.

Numerous studies have been conducted to reconstruct accidents and to do the analysis to prevent accident. Based-on research [10][11], it is stated that Fuzzy Logic becomes an alternative method for classifying crash data taken from the accelerometer and OBD II in consideration to that this method uses a fairly light computational model compared to the learning model. Forensic analysis for vehicle crash reconstruction using a numerical modeling approach can

Cite: Erfianto, B., \& Rahmatsyah, A. (2021). Forensic Analysis of Braking Classification Based on Acceleration, Jerk, and Velocity Data. Kinetik: Game Technology, Information System, Computer Network, Computing, Electronics, and Control, 6(3). https://doi.org/10.22219/kinetik.v6i3.1284 
predict vehicle behavior and reduce the need for real-scale crash tests. Of the modeling approaches, a centered parameter model (LPM) and a finite element model (FEM) have been used in the reconstruction of such vehicle accidents [12]. While in research [13][14][15][16] it was proposed an erratic driving detection system generally using Fuzzy Logic and Artificial Intelligence. In general, the system retrieves data from the 3-axis accelerometer, camera, and on-board diagnostic reader (OBD-II), which provides a reading of the vehicle speed. The system output refers to a level of driving risk in the range of normal to dangerous. Regarding OBD II recorded data, [5] proposes three levels of comfort: detection of the peak acceleration threshold; threshold detection-jerk based on the derivative of the acceleration (jerk); and comfort rating of acceleration according to ISO2631-1 standard.

One of vehicle data mostly analyzed for forensic purposes is braking data, which are influenced by the condition of the driver's behaviour. Vehicle braking is a complex process determined by many factors, such as vehicle condition, road construction, and mental and physiological condition of driver, which must be assessed to describe the detailed braking condition, especially for the forensic analysis purpose [17]. Dapzol [18] for instance, pioneered the use of the Hidden Markov Model as an adaptive system to determine or predict driver behaviour using vehicle braking data. The braking distance is mostly determined by vehicle speed before braking, response time of driver, deceleration time, variation time of deceleration (generally called as jerk) [19].

Groot [20] defines several hazardous situation classifications depending on the type of maneuver being performed by the driver, the severity of the situation. Meanwhile, Cheng [21] uses a braking classification based on near-crashes, crash relevant events, and proximity events, where near-crashes have the highest severity near-crashes. In this paper, the classification of braking used is based on Wu [26], which is comfortable, discomfort and dangerous. Wu uses the terminology car following model where the distance between two cars must always be greater than zero, so that braking can be done to avoid collisions. This research is then used as a reference for the classification of braking, including [22][23][24][25].

Wu in his research [26] used a safe and comfortable braking model that we must do with an assumption that the car stops before the crash occurs. The testing results showed the minimum acceleration enabling the passengers to be uncomfortable at $0.25 \mathrm{~g}$, and if the longitudinal acceleration is greater than $0.5 \mathrm{~g}$, the passenger will find it difficult to stand up. Passengers commonly should not be worried as longitudinal acceleration is commonly lower than $0.1 \mathrm{~g}$ and it can only reach a maximum value of $0.3 \mathrm{~g}$. Although it is uncertain which limit of stable acceleration is acceptable, the upper limit for normal operation seems to be approximately $2.0 \mathrm{~m} / \mathrm{s}^{2}$.

Based on the related studies as presented in the paragraphs above, the problem focused in this research paper is how to use deceleration, speed and jerk to categorize the braking type in a vehicle based upon the forensic analysis. In the early phase, the collection of data for acceleration and speed has been carried out using the on-board device on a vehicle, and an algorithm has been developed to perform forensic analysis based on the collected data. In this paper, forensic analysis was limited to produce a braking categorization based upon the data gathered. The contribution of this paper is to propose a braking detection model by combining the data of acceleration, speed and jerk in an algorithm based on Fuzzy Inference System. The evaluation of this model is presented using data taken from the MPV vehicle. The results of a forensic analysis of braking data can offer a better general understanding of the braking maneuvers themselves, which can be further developed to identify one of the accident factors and to provide some recommendations on which actions to include in future analysis.

\section{Research Method}

\subsection{Overview of Digital Forensic Analysis}

A simplified block diagram of vehicle forensic analysis system based on braking data is depicted in Figure 1 . The raw signal is obtained from the SD card memory attached to the vehicle data recorder. Accelerometer data is taken from ADXL345, while speed data is recorded from OBD II port. Both data are then recorded onto the MicroSD card in the prototype vehicle data recorder.

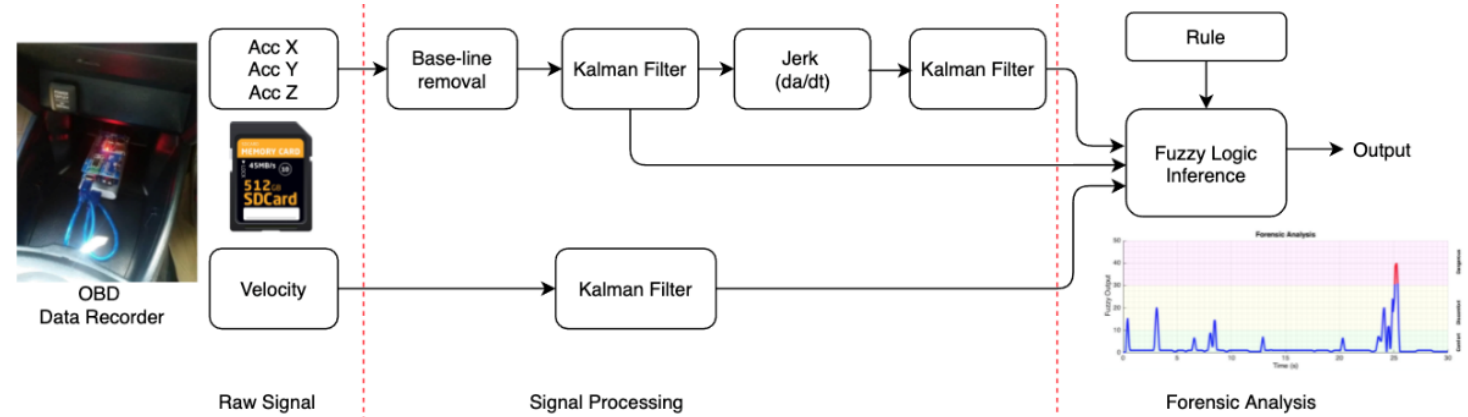

Figure 1. Block Diagram of System

(C) 2021 The Authors. Published by Universitas Muhammadiyah Malang

This is an open access article under the CC BY SA license. (https://creativecommons.org/licenses/by-sa/4.0/) 
The signal processing block functions to perform the initial base-line or DC component removal of the accelerometer signal. Furthermore, the Kalman Filer block functions to perform noise removal from ac- celerometer and velocity signal. The results of the filtered velocity signal are then used as input for the Fuzzy inference system. This paper uses a jerk signal derived from the accelerometer. Jerk or jerk is a derivative of acceleration and has been used by many researchers to measure driving behavior [27][28]. Jerk is directly proportional to the time of force change and inversely proportional to mass. Therefore, the discomfort of driv- ing can increases whenever there is a high longitudinal deceleration (high negative acceleration) triggered by sudden, or emergency braking [29].

The computation of the rate of acceleration change, known as a jerk, is carried out simultaneously using the differentiation method. Then, the method proposed in this paper is to use acceleration, velocity, and jerk as inputs for the Fuzzy inference system to perform forensic analysis of braking conditions. As the results, the status of a vehicle can be obtained in terms of driver behavior in terms of braking, whether it is comfort, discomfort, or dangerous based on [22]. The following paragraph describes the parts of the block diagram as shown in Figure 1.

\subsection{Data Acquisition}

An event data recorder prototype has been built to collect data, called a VDR (vehicle data recorder). VDR retrieves data from the ECU (engine control unit) via the OBD II port with CAN protocol. Basically, many IDs of vehicle sensors can be accessed for data acquisition. However, we only use Velocity ID for braking forensic analysis. Since ECU and OBD II systems themselves do not provide IMU internally, the accelerometer in the VDR functions to retrieve acceleration data, in this case, the $x, y$ and $z$ axes of the accelerometer. All accelerometer data is stored in the MicroSD as evident data for forensic analysis. The PCB design for the VDR circuit can be seen in Figure 2. The placement of the VDR board device on a vehicle or car can be seen on the 2 on the right. Data collection is based on experiments using MPV vehicles.

The VDR continuously monitors the acceleration changes and records the signal to the MicroSD mem- ory for a certain period before and after the braking process. The VDR consists of several components, such as IMU ADXL345, in which the accelerometer and data acquisition unit are the main parts with a resolution of $0.01 \mathrm{~g}$. The accelerometer is set to use a measurement range of $2 \mathrm{~g}$. The accelerometer can measure dynamic acceleration during brake maneuver, with an internal sampling frequency of $50 \mathrm{~Hz}$.
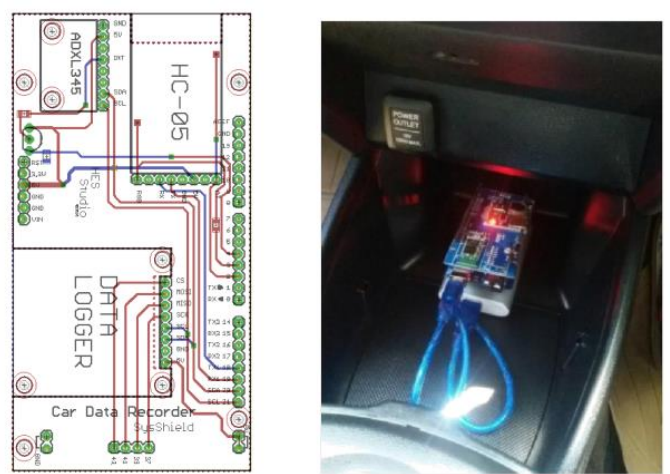

Figure 2. Left: PCB Design Layout of VDR. Right: Installation of VDR in Vehicle Dashboard

\subsection{Signal Processing}

The data are taken from the accelerometer and the OBD II port still contains much noise. Thus, it will significantly affect the results of the forensic analysis. The noise is in the form of high frequency and also baseline noise. For this reason, filtering is carried out to remove unnecessary noise in the data. Based on Figure 1, the first thing to do is how to remove the DC component so that the accelerometer signal moves around the 0 of $Y$-axis. This process is known as baseline removal. This paper uses a simple FIR in Equation 1 [30] which removes the mean of all signal samples.

$$
\widehat{\operatorname{acc}}[m]=\operatorname{acc}[m]-\frac{1}{N} \sum_{n=0}^{N-1} \operatorname{acc}[n]
$$

Where $\widehat{a c c}[\mathrm{~m}]$ is the accelerometer value after baseline removal, acc $[\mathrm{m}]$ is the current accelerometer value, and $N$ is the length of data. and the last term is the mean of the accelerometer data block. The results of the baseline removal can be seen in Figure 3. 


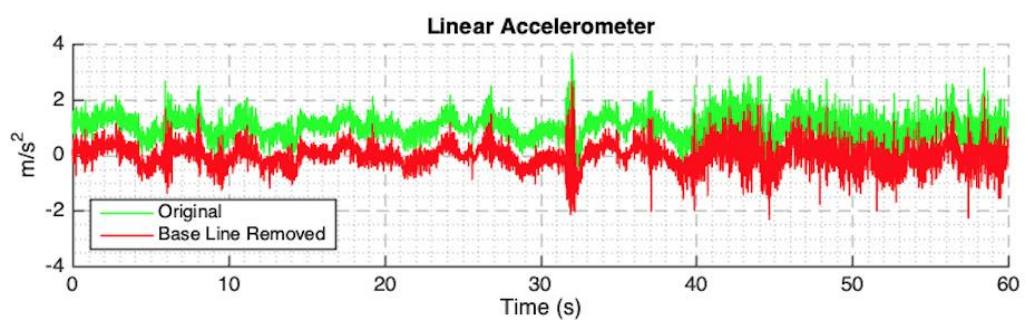

Figure 3. Original Signal and After Baseline Removal of accelerometer Signal

After eliminating the baseline signal or DC component, the following process is to use the Kalman filter for smoothing the accelerometer signal, velocity, and jerk. Theoretically in Equation 2 [27], a jerk signal is obtained by differentiating the value of the accelerometer.

$$
j\left(\frac{m}{s^{3}}\right)=\frac{d(a c c)}{d t}
$$

Regarding Algorithm 1, the Kalman Filter algorithm is used to remove noise from the accelerometer signal, speed, and jerk. In short, the Kalman Filter algorithm that is used to remove noise on the x-axis accelerometer signal is described in line 5-10 of Algorithm 1. The implementation of Kalman filter algorithm also applies to all accelerometer axis, jerk signals, and velocity signals by changing raw signals variable assignment in line 2 of Algorithm 1.

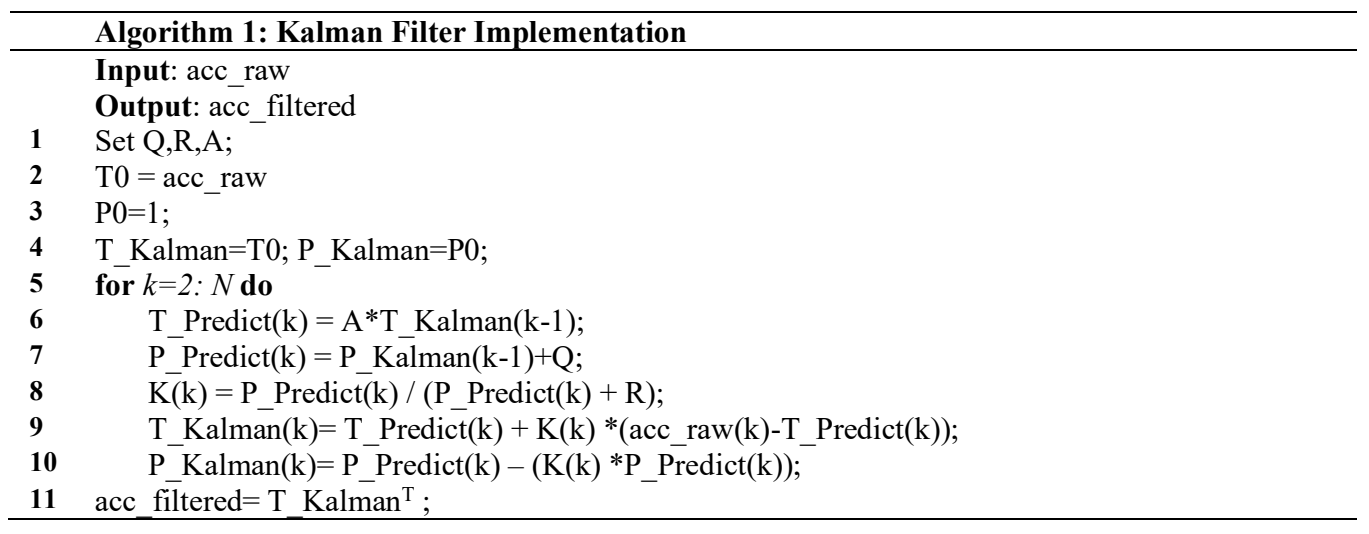

Figure 4 is an example of an accelerometer signal, velocity, and jerk that still contains noise (red signals), while Kalman Filter filters the blue signals. After the noise removal is done, the accelerometer signals, velocity, and jerk signals are fetched into the Fuzzy inference system.
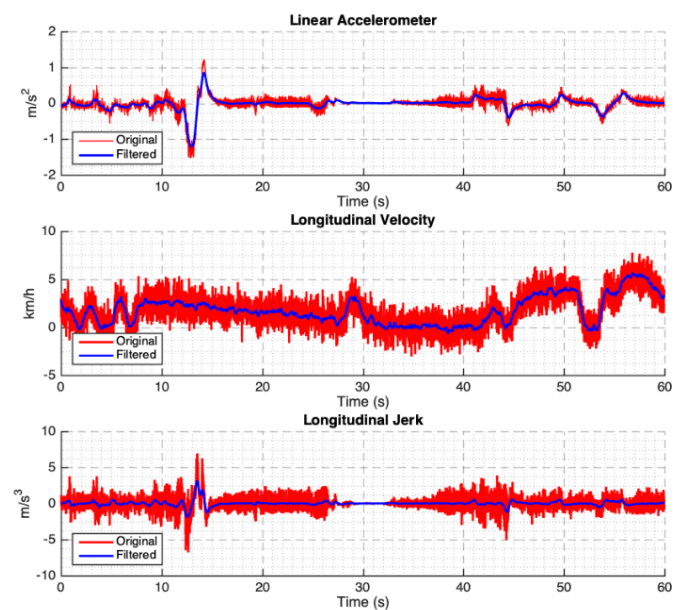
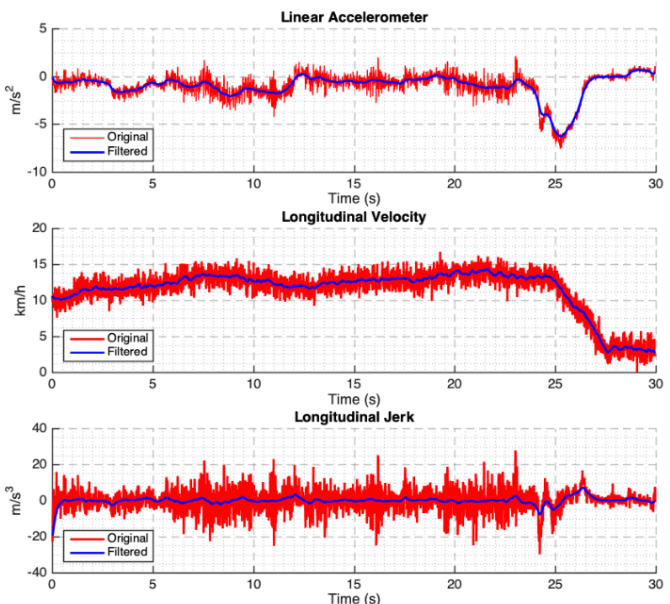

Figure 4. Raw Signals of Acceleration and Velocity. Jerk Signal Obtained by Differentiating Acceleration Signals. Left: The Braking Condition when the Road is in a Jam. Right: Emergency Braking Condition

(C) 2021 The Authors. Published by Universitas Muhammadiyah Malang

This is an open access article under the CC BY SA license. (https://creativecommons.org/licenses/by-sa/4.0/) 


\subsection{Fuzzy Inference System Design}

The driver's behavior affects the deceleration, braking distance, and braking time completed by various vehicles. The driver takes more distance to slow down the vehicle in ideal conditions if the velocity is high. The speed at which the driver reaches maximum deceleration (called critical speed) also depends on various vehicles. It also implies that at a higher velocity, the driver reaches the maximum deceleration quickly. The results of this study can be used to enter the values of acceleration, deceleration, and velocity of the braking classification model [31]. The Institute for Traffic Engineering's recommends average decelerating rate is $3.0 \mathrm{~m} / \mathrm{s} 2$ [32] and ac- cording to AASHTO [33] is $3.4 \mathrm{~m} / \mathrm{s} 2$ for four wheels vehicles. In this paper, the average maximum deceleration rate observed for gasoline cars is $3.88 \mathrm{~m} / \mathrm{s}$ and for diesel cars is $4.53 \mathrm{~m} / \mathrm{s}$. Zeeman [34] defines a longitudinal deceleration with the rate of $3.5 \mathrm{~m} / \mathrm{s}$. It is equivalent to $0.35 \mathrm{~g}$ as the ideal deceleration for a highway stop. This value is a comfortable level of retarding (according to the [22] classification) for most drivers in maintaining steering control during braking maneuvers.

Bagdadi and Varhelyi [17] show that for a situation where the initial speed is at $50 \mathrm{~km} / \mathrm{h}$ and $70 \mathrm{~km} / \mathrm{h}$, it has about 1.5 seconds to perform the braking maneuver, the average rate of change in acceleration is $1 \mathrm{~g} / \mathrm{s}$ or about $9.81 \mathrm{~m} / \mathrm{s}$. Thus, in this paper, a value of $1 \mathrm{~g} / \mathrm{s}$ was chosen as the maximum threshold for hazardous braking rates. Naude [35] states that the speed of the vehicle when the driver starts braking with the vehicle uses ABS (Anti-lock Brake System) and BAS (Brake Assistance System). The braking distance is approximately $30 \mathrm{~m}$, and the average deceleration is about $7.6 \mathrm{~m} / \mathrm{s}$, with a maximum peak at $11 \mathrm{~m} / \mathrm{s}$. Based on the deceleration, velocity, and jerk value as mentioned in the reference, we further define the membership function of Fuzzy logic as depicted in Table 1.

The second stage of designing the Fuzzy inference system is creating a rule-based on each parameter's data and boundary values. The rule design is created using the Matlab Fuzzy Toolbox application. In addition, we can define rules as much as possible. Figure 5 depicts rules definition that is made to find out the final result of each data obtained. Since three variables are used as input with three membership functions, the result Fuzzy inference system is also depicted in the right of Figure 5. Generally, it is possible to add some rules if needed. In Figure 5, one of the data is input into the rules that have been made, so the rule design shows whether the forensic braking data shows comfort, discomfort, or dangerous. Since the limited space in this paper, the Fuzzy inference system design process will not be discussed in detail in this paper.

Table 1. Fuzzy Membership Function

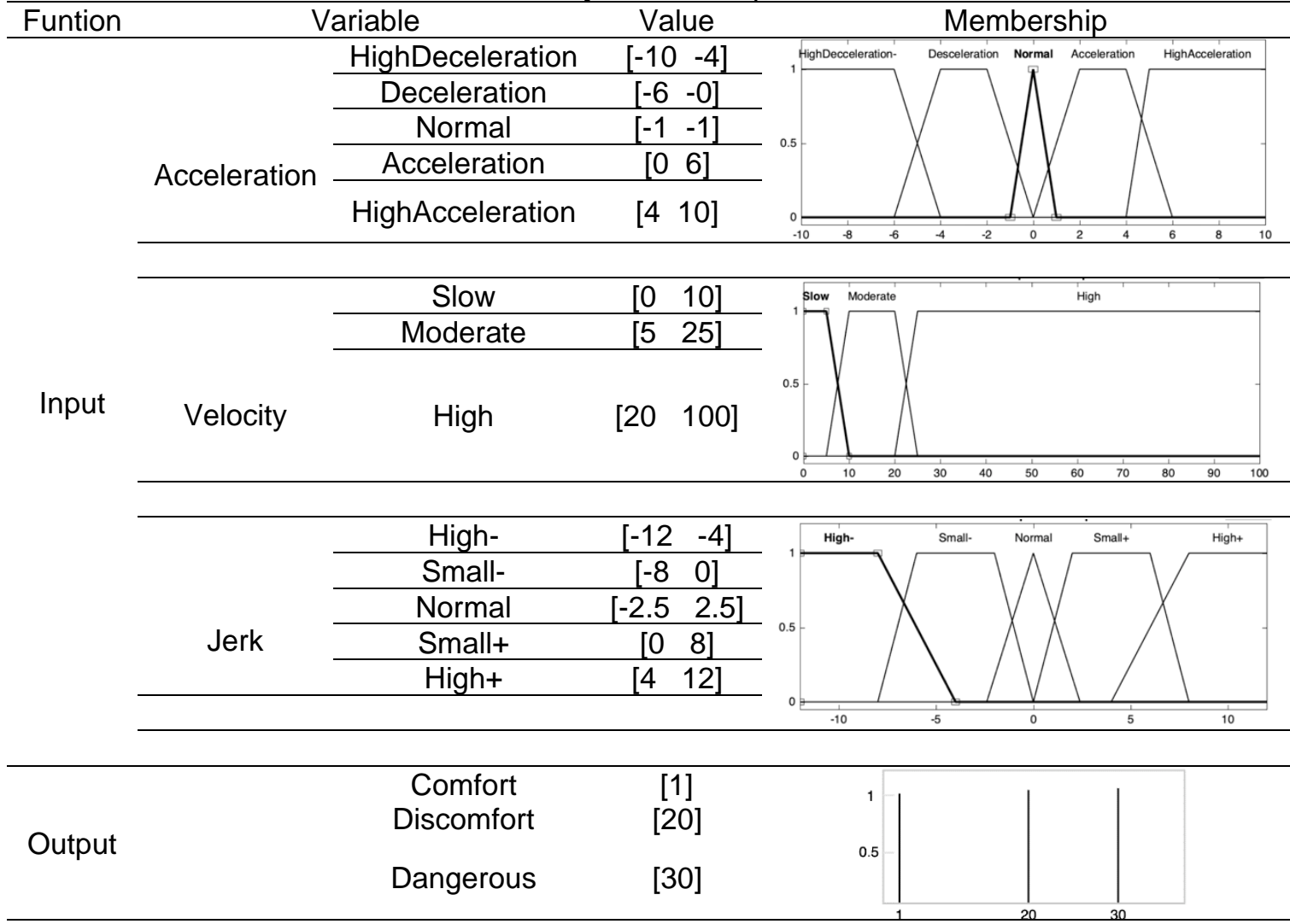



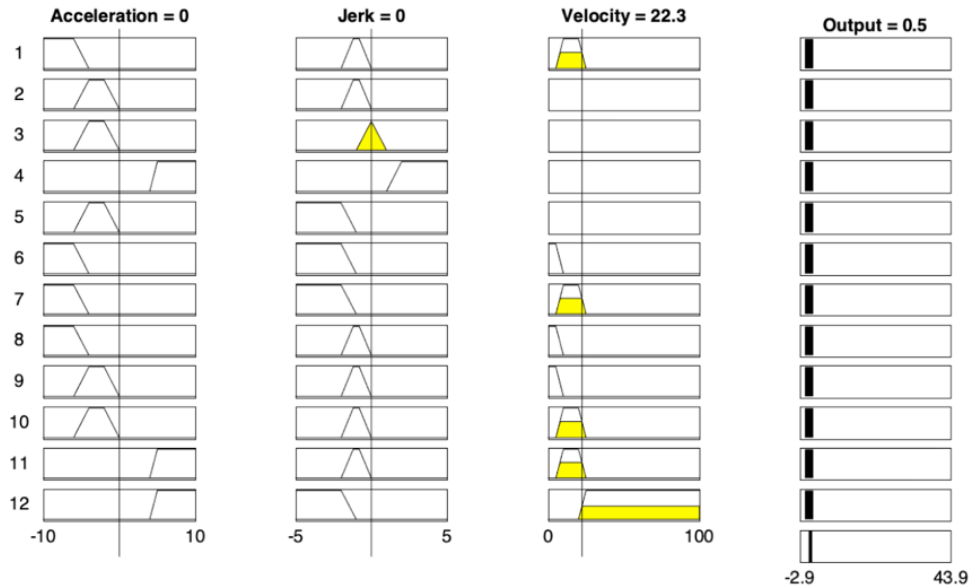

Figure 5. The Simulation of Fuzzy Inference Rule and the Fuzzy Output Showing the Category of the Results of the Braking Condition Forensic

\section{Results and Discussion}

\subsection{Deceleration and Velocity}

The plot of acceleration for each driving condition and speed value can be seen in Figure 6. As mentioned in the previous paragraph, acceleration and velocity are obtained from the vehicle data recorder. For the experiment purposes, acceleration (in braking condition it is called deceleration) data is collected on Jakarta-Cikampek Toll Road and in Bandung City (normal conditions and traffic jams). Especially for recording sudden and dangerous braking conditions, data collection was collected around the GBLA Bandung Stadium during no traffic condition. Vehicle speed varies according to different types of driving. The average driving speed on inner-city roads and toll roads is maintained at 30 $\mathrm{km} / \mathrm{h}$ to $60 \mathrm{~km} / \mathrm{h}$. During a traffic jam on inner-city road, the vehicle's speed is detected at $5 \mathrm{~km} / \mathrm{h}$ to $15 \mathrm{~km} / \mathrm{h}$.
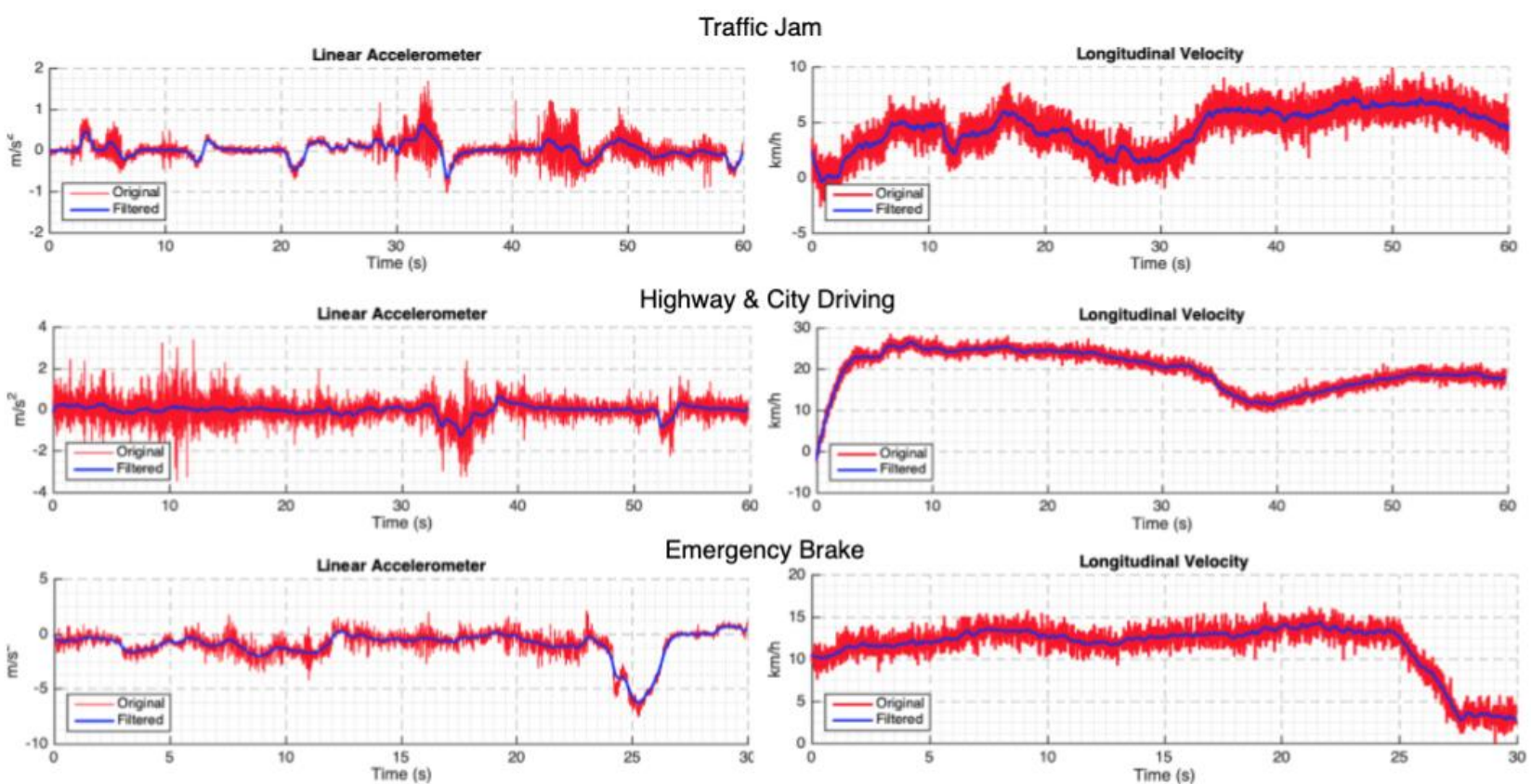

Figure 6. Acceleration and Velocity from Various Driving Condition

During emergency brake, the vehicle ecperiences deceleration significantly. Since there exist inertial force, however, the vehicle does not stop suddenly when the emergency brake is executed. This condition is depicted in Figure 6 below. For forensic analysis purposes, the plot in Figure 6 shows a weakness if only the threshold of accelerometer and velocity are used. Therefore, in this proposed forensic analysis model, the jerk values are used for various types of driving to extract the information wether the braking system is dangerous of not due to the derivative of acceleration. 


\subsection{Analysis of Braking Data: City and Toll Roads}

This section describes the example of forensic analysis of braking when the vehicle runs on toll roads, inner-city roads, and normal and when traffic is a jam. The purpose of collecting data at different driving locations is to obtain braking patterns, which will later be categorized into comfort, discomfort, and dangerous braking, referring to [22]. Examples of braking data and the results of a forensic analysis can be seen in Figure 7.

The left side of Figure 7 shows data input for forensic analysis. The vehicle's velocity while driving on toll roads and inner-city roads is in the range of $15 \mathrm{~km} / \mathrm{hr}$ to $20 \mathrm{~km} / \mathrm{hour}$. The data is collected around 9.00 to $10.00 \mathrm{WIB}$ in the morning; the traffic is dense but not categorized as a traffic jam. The resulting deceleration or jerk data does not appear to be significant or is still under the category of [22]. Thus, the forensic analysis concluded that in the crowded condition, the braking occurred several times but still in the comfort category indicated by $100 \%$ of comfort data.

For travelling in a traffic jam, the vehicle's speed is around $5 \mathrm{~km} / \mathrm{h}-10 \mathrm{~km} / \mathrm{h}$. Accelerometer data shows deceleration with a recurring frequency, given the road is in traffic conditions and the driver brakes frequently. Jerk data derived from deceleration still shows a value below the threshold. Thus, based on forensic analysis, it can be concluded that there are several brakes in the city during traffic jams, but still in the comfort data $96.55 \%$ and discomfort data $3.36 \%$.

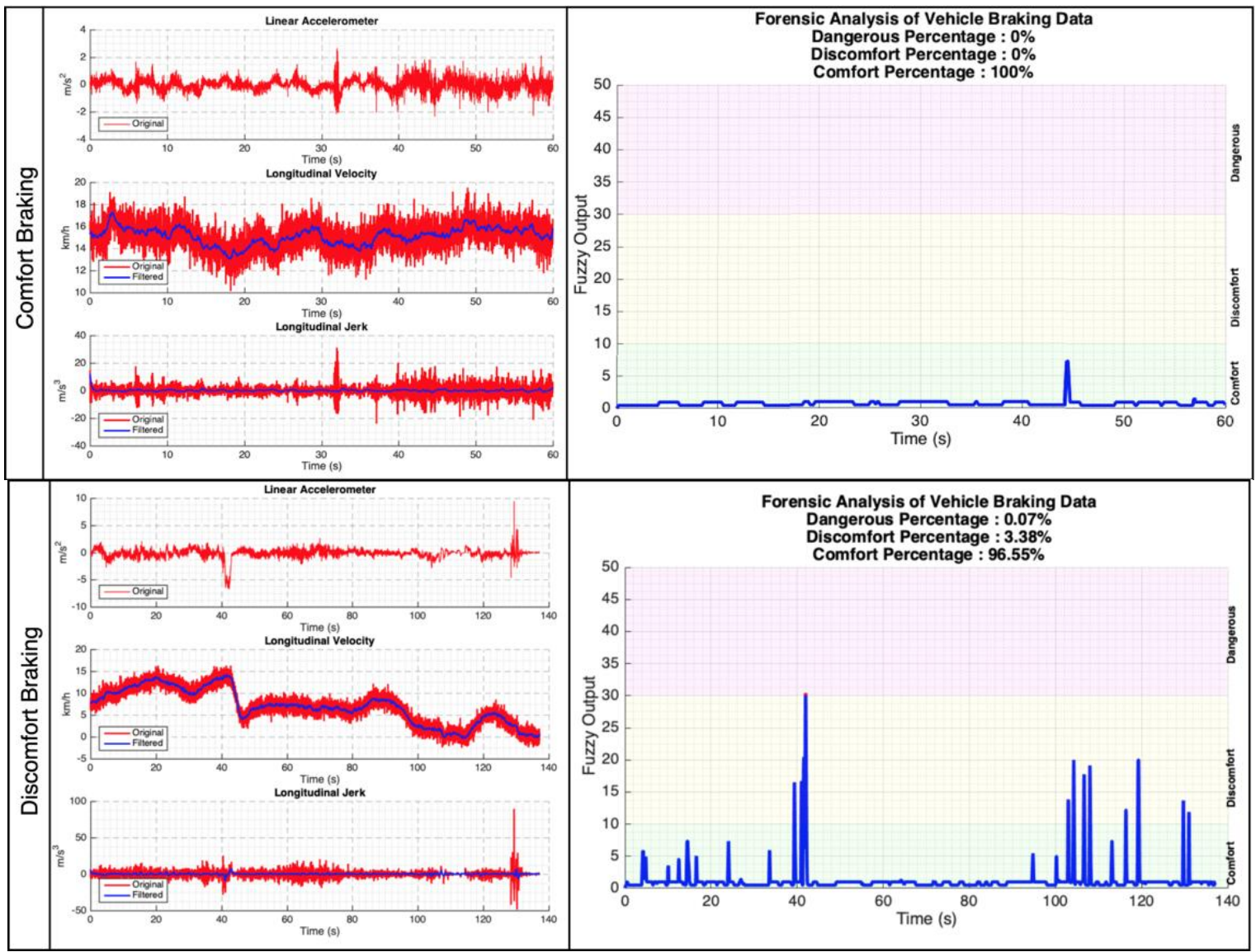

Figure 7. Fragment Data that Shows the Results of Forensic Analysis of Braking Data. Above: Results with $100 \%$ Comfort Braking. Below: Results that Contains 0.07\% Dangerous, 3.38\% Discomfort, and 96.55\% Comfort Braking

\subsection{Analysis of Braking Data: Dangerous Category}

This part explains the results of the braking forensic analysis by presenting three categories of brake as shown in Figure 8. For the case of braking with comfort category, the recording data showed the deceleration reaching $-2 \mathrm{~m} / \mathrm{s} 2$ determined by the effect of braking. Though the data of speed showed a drastic decrease within $1 \mathrm{~s}$ to $2 \mathrm{~s}$, the effect of jerk was not so significant; therefore, the Fuzzy inference system stated that the braking forensic data was in the comfort category with the output of Fuzzy <10. A different condition occurred in the Discomfort braking, i.e., when the decrease of speed was quite drastical and in a period that was quite frequent accompanied with the significantly large value of deceleration, i.e., approximately $-5 \mathrm{~m} / \mathrm{s} 2$ with the jerk value approaching $-10 \mathrm{~m} / \mathrm{s}^{3}$. 


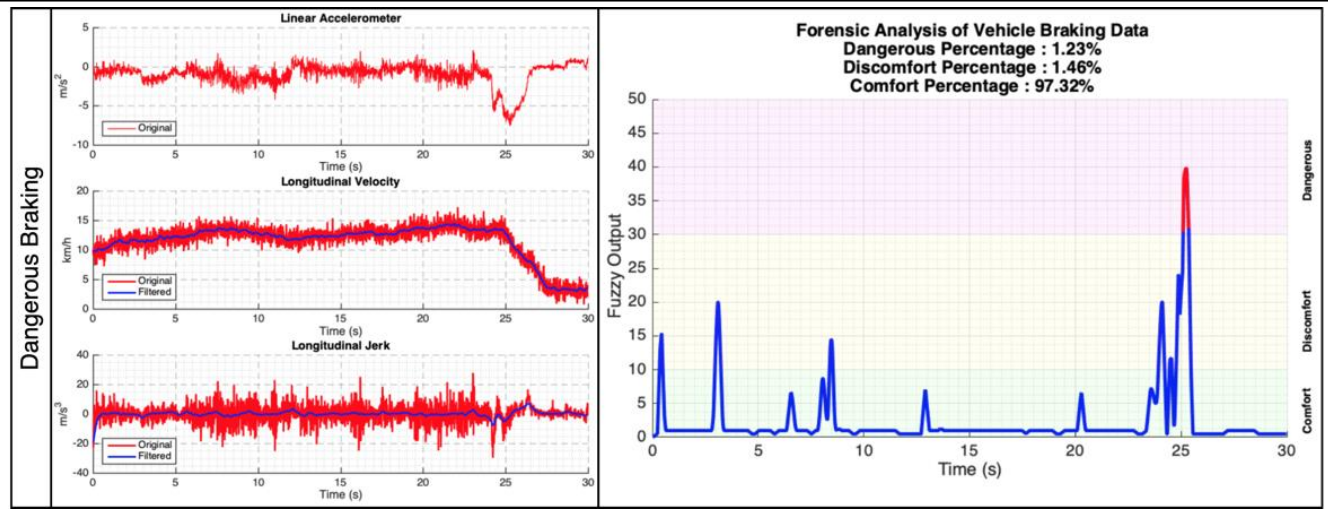

Figure 8. Fragment Data that Shows the Results of Forensic Analysis of 1.23\% dangerous Braking

In Figure 8 in the last part, the forensic analysis concluded that there has been a braking categorized in dangerous braking. The forensic data of deceleration showed a quite significant value, i.e., approximately $-8 \mathrm{~m} / \mathrm{s} 2$. The vehicle speed also experienced the decrease within approximately $2.5 \mathrm{~s}$. The braking condition also created a quite high jerk around $12 \mathrm{~m} / \mathrm{s} 3$ (see remark 1 in Figure 8). The high jerk was remarked with the forward jerk felt by the driver. Based upon the values obtained, the forensic analysis based on the Fuzzy inference system concluded that there has been a dangerous braking condition between 25 and 26 seconds. This can be seen in the part of output Fuzzy logic as remarked by the red plot. Certainly, the results of Fuzzy inference system-based analysis can be used further as forensic evidence, for instance, to determine when the accident (or crash) occurs, and whether the driver does a brake (braking effect). Also, other investigation question can be developed. For the further development, it not simply uses the forensic data of acceleration, speed and jerk. Other data needs to be integrated into the system to result in a quite strong forensic conclusion to see the factor of an accident.

\section{Conclusion}

For the forensic analysis purpose, most of previous researchers used acceleration / deceleration and speed for the detection system of driver's attitude. This paper presents a forensic analysis model using deceleration, velocity and jerk data as velocity derivatives. The forensic data were then processed using an algorithm based on the Fuzzy inference system that we built to categorize the vehicle braking condition, whether they were comfort, discomfort, or dangerous category. One of the contributions of this paper is the longitudinal acceleration (in this case more specifically as deceleration) along with the jerk caused by changes in deceleration with time in which it can be used as forensic evidence of the braking condition of the drivers. Forensic data can show between driving in urban environments and driving on highway in terms of normal and hazardous braking categories. Although the value of each variable varied with different driving situations, this can be adapted to certain environments, such as the vehicle type and the speed category used. From the experiments conducted, it showed that the model proposed in this paper can be used as a reference as a computational model for processing braking data and producing forensic decisions, for example, used to find factors causing crash or accidents. Further, the use of other sensors, taken from OBD or external sensors, can strengthen forensic analysis decisions to find the cause of an accident.

\section{Acknowledgment}

This is funded by Hibah Penelitian Strategis Nasional Institusi RISTEKDIKTI of 2018-2019. The author would like to thank the team helping to design the prototype vehicle data recorder and data collection, including: Satrio Adi Nugroho, Aini Rahmawati, Intan Rizky, and Ihsan Ade.

\section{References}

[1] V. Dobromirov, S. Dotsenko, V. Verstov, and S. Volkov, "Methods of examining vehicle electronic systems in the course of automotive forensic expert examinations," Transportation Research Procedia, vol. 20, pp. 143-150, 2017. https://doi.org/10.1016/j.trpro.2017.01.037

[2] D. Connolly, "Event data recorder as a forensic tool," in Proceedings of the ITRN, 2014.

[3] T. Hoppe, S. Kuhlmann, S. Kiltz, and J. Dittmann, "IT-forensic automotive investigations on the example of route reconstruction on automotive system and communication data," in International Conference on Computer Safety, Reliability, and Security. Springer, 2012, pp. 125-136. https://doi.org/10.1007/978-3-642-33678-2_11

[4] J. S. Daily, N. Singleton, E. Downing, and G. W. Manes, "The forensics aspects of event data recorders," Journal of Digital Forensics, Security and Law, vol. 3, no. 3, p. 2, 2008. https://doi.org/10.15394/jdfsl.2008.1044

[5] J. C. Castellanos, A. A. Susin, and F. Fruett, "Embedded sensor system and techniques to evaluate the comfort in public transportation," in 2011 14th International IEEE Conference on Intelligent Transportation Systems (ITSC). IEEE, 2011, pp. 1858-1863.https://doi.org/ 10.1109/ITSC.2011.6083051

[6] D. Le Nguyen, M.-E. Lee, and A. Lensky, "The design and implementation of new vehicle black box using the obd information," in Computing and Convergence Technology (ICCCT), 2012 7th International Conference on. IEEE, 2012, pp. 1281-1284.

(c) 2021 The Authors. Published by Universitas Muhammadiyah Malang

This is an open access article under the CC BY SA license. (https://creativecommons.org/licenses/by-sa/4.0/) 
[7] A. X. A. Sim and B. Sitohang, "OBD-II standard car engine diagnostic software development," in Data and Software Engineering (ICODSE), 2014 International Conference on. IEEE, 2014, pp. 1-5. https://doi.org/10.1109/ICODSE.2014.7062704

[8] J.-S. Jhou, S.-H. Chen, W.-D. Tsay, and M.-C. Lai, "The implementation of obd-ii vehicle diagnosis system integrated with cloud computation technology," in Robot, Vision and Signal Processing (RVSP), 2013 Second International Conference on. IEEE, 2013, pp. 9-12. http://doi.org/10.1109/RVSP.2013.55

[9] S. G. R. J. Naieni, A. Makui, and R. Ghousi, "An approach for accident forecasting using fuzzy logic rules: A case mining of lift truck accident forecasting in one of the Iranian car manufacturers," International Journal of Industrial Engineering, vol. 23, no. 1, pp. 53-64, 2012.

[10] B. B. Munyazikwiye, H. R. Karimi, and K. G. Robbersmyr, "Fuzzy logic approach to predict vehicle crash severity from acceleration data," in Fuzzy Theory and Its Applications (iFUZZY), 2015 International Conference on. IEEE, 2015, pp. 44-49. https://doi.org/10.1109/iFUZZY.2015.7391892

[11] A. Fasanmade, Y. He, A. H. Al-Bayatti, J. N. Morden, S. O. Aliyu, A. S. Alfakeeh, and A. O. Alsayed, "A fuzzy-logic approach to dynamic bayesian severity level classification of driver distraction using image recognition," IEEE Access, vol. 8, pp. $95197-95207,2020$. https://doi.org/10.1109/ACCESS.2020.2994811

[12] B. B. Munyazikwiye, "Mathematical modelling and analysis of vehicle frontal crash using lumped parameters models," 2020.

[13] A. Aljaafreh, N. Alshabatat, and M. S. N. Al-Din, "Driving style recognition using fuzzy logic," in 2012 IEEE International Conference on Vehicular Electronics and Safety (ICVES 2012). IEEE, 2012, pp. 460-463. https://doi.org/10.1109/ICVES.2012.6294318

[14] M. Santos and V. Lo pez, "Fuzzy decision system for safety on roads," in Handbook on Decision Making. Springer, 2012, pp. 171-187. https://doi.org/10.1007/978-3-642-25755-1 9

[15] Z. Halim, R. Kalsoom, S. Bashir, and G. Abbas, "Artificial intelligence techniques for driving safety and vehicle crash prediction," Artificial Intelligence Review, vol. 46, no. 3, pp. 351-387, 2016. https://doi.org/10.1007/s10462-016-9467-9

[16] I. S. Feraud, M. M. Lara, and J. E. Naranjo, "A fuzzy logic model to estimate safe driving behavior based on traffic violation," in 2017 IEEE Second Ecuador Technical Chapters Meeting (ETCM). IEEE, 2017, pp. 1-6. https://doi.org/ 10.1109/ETCM.2017.8247536

[17] O. Bagdadi, "Assessing safety critical braking events in naturalistic driving studies," Transportation re- search part F: traffic psychology and behaviour, vol. 16, pp. 117-126, 2013. https://doi.org/10.1016/j.trf.2012.08.006

[18] N. Dapzol, "Drivers behaviour modelling using the hidden markov model formalism," in ECTRI Young researchers seminar, The Hague, the Netherlands, vol. 2, no. 2.2, 2005, pp. 2-1.

[19] N. Kudarauskas, "Analysis of emergency braking of a vehicle," Transport, vol. 22, no. 3, pp. $154-159,2007$. https://doi.org/10.1080/16484142.2007.9638118

[20] S. DeGroot, J.DeWinter, P.Wieringa, and M.Mulder, "An analysis of braking measures,"in Proceedings Driving Simulation Conference 2009, Monaco, 2010

[21] B. Cheng, Q. Lin, T. Song, Y. Cui, L. Wang, and S. Kuzumaki, "Analysis of driver brake operation in near-crash situation using naturalistic driving data," International Journal of Automotive Engineering, vol. 2, no. 4, pp. 87-94, 2011. https://doi.org/10.20485/jsaeijae.2.4_87

[22] Z. Wu, Y. Liu, and G. Pan, "A smart car control model for brake comfort based on car following," IEEE transactions on intelligent transportation systems, vol. 10, no. 1, pp. 42-46, 2008. https://doi.org/ 10.1109/TITS.2008.2006777

[23] X. Xiang, W. Qin, and B. Xiang, "Research on a dsrc-based rear-end collision warning model," IEEE Transactions on Intelligent Transportation Systems, vol. 15, no. 3, pp. 1054-1065, 2014.https://doi.org/10.1109/TITS.2013.2293771

[24] S. Huo, L. Yu, L. Ma, and L. Zhang, "Ride comfort improvement in post-braking phase using active suspension," in International Design Engineering Technical Conferences and Computers and Information in Engineering Conference, vol. 57168. American Society of Mechanical Engineers, 2015, p. V006T10A072. https://doi.org/10.1115/DETC2015-46878

[25] S. P. Deligianni, M. Quddus, A. Morris, A. Anvuur, and S. Reed, "Analyzing and modeling drivers deceleration behavior from normal driving," Transportation research record, vol. 2663, no. 1, pp. 134-141, 2017. https://doi.org/10.3141/2663-17

[26] H. Bellem, B. Thiel, M. Schrauf, and J. F. Krems, "Comfort in automated driving: An analysis of pref- erences for different automated driving styles and their dependence on personality traits," Transportation research part F: traffic psychology and behaviour, vol. 55, pp. 90-100, 2018. https://doi.org/10.1016/j.trf.2018.02.036

[27] O. Bagdadi and A. Varhelyi, "Jerky drivingan indicator of accident proneness?" Accident Analysis \& Prevention, vol. 43, no. 4, pp. 1359-1363, 2011. https://doi.org/10.1016/j.aap.2011.02.009

[28] O. Bagdadi and A. Varhelyi ,"Development of a method for detecting jerks in safety critical events," Accident Analysis \& Pre- vention, vol. 50, pp. 83-91, 2013. https://doi.org/10.1016/j.aap.2012.03.032

[29] J. Cao, H. Lu, K. Guo, and J. Zhang, "A driver modeling based on the preview-follower theory and the jerky dynamics," Mathematical Problems in Engineering, vol. 2013, 2013. https://doi.org/10.1155/2013/952106

[30] Pan, Chao and Zhang, Ruifu and Luo, Hao and Shen, Hua," Baseline correction of vibration acceleration signals with inconsistent initial velocity and displacement, "Advances in Mechanical Engineering, vol. 8, no 10, 2016. https://doi.org/10.1177/1687814016675534

[31] P. S. Bokare and A. K. Maurya, "Acceleration-deceleration behaviour of various vehicle types," Trans- portation research procedia, vol. 25, pp. 4733-4749, 2017. https://doi.org/10.1016/j.trpro.2017.05.486

[32] B. Wolshon, A. Pande et al., Traffic engineering handbook. John Wiley \& Sons, 2016.

[33] A.AASHTO,"Policy on geometric design of highways and streets,"American Association of State Highway and Transportation Officials, Washington, DC, vol. 1, no. 990, p. 158, 2001.

[34] A. S. Zeeman and M. J. Booysen, "Combining speed and acceleration to detect reckless driving in the informal public transport industry," in 16th International IEEE Conference on Intelligent Transportation Systems (ITSC 2013). IEEE, 2013, pp. 756-761. https://doi.org/10.1109/ITSC.2013.6728322

[35] C. Naude, T. Serre, M. Dubois-Lounis, J.-Y. Fournier, D. Lechner, M. Guilbot, and V. Ledoux, "Acquisition and analysis of road incidents based on vehicle dynamics," Accident Analysis \& Prevention, vol. 130, pp. 117-124, 2019. https://doi.org/10.1016/j.aap.2017.02.021

Cite: Erfianto, B., \& Rahmatsyah, A. (2021). Forensic Analysis of Braking Classification Based on Acceleration, Jerk, and Velocity Data. Kinetik: Game Technology, Information System, Computer Network, Computing, Electronics, and Control, 6(3). https://doi.org/10.22219/kinetik.v6i3.1284 
